Original article

\title{
Reliability of measuring the circumference, surface area, and volume of a body part or an object using a three-dimensional scanner
}

\author{
Toshihiro Kawae $^{1 *}$, Yuki Nakashima ${ }^{2}$, Daisuke Iwaki ${ }^{2}$, Naoya Goto ${ }^{2}$, Kenichi Fudeyasu ${ }^{2}$, \\ Tomoyasu Ishiguro ${ }^{1}$, Kiyokazu Sekikawa ${ }^{3}$, Yasutaka Umayahara ${ }^{4}$, Hiroaki Kimura ${ }^{5}$ \\ ${ }^{I}$ Makuhari Human Care Faculty Department of Physical Therapy, Tohto University \\ ${ }^{2}$ Division of Rehabilitation, Department of Clinical Practice and Support, Hiroshima University Hospital \\ ${ }^{3}$ Department of Physical Analysis and Therapeutic Sciences, Graduate School of Biomedical and Health Sciences, Hiroshima University \\ ${ }^{4}$ Department of Rehabilitation, Faculty of Health Sciences, Hiroshima Cosmopolitan University \\ ${ }^{5}$ Department of Rehabilitation, Hiroshima University Hospital
}

\section{ABSTRACT}

Introduction: In recent years, small and lightweight three-dimensional (3D) scanners have been used to measure circumference, surface area, and volume of the body or objects; however, their measurement reliability remains unclear. Therefore, this study aimed to clarify the reliability of measuring the circumference, surface area, and volume of a body part or an object using the handheld 3D scanner.

Methods: Regarding the intra-rater reliability, one examiner measured and calculated the circumference, surface area, and volume of the upper arm of three participants. Regarding the inter-rater reliability, three objects were scanned by three different examiners, and the circumference, surface area, and volume were calculated. Intra-class correlation coefficients (ICC) were used to evaluate the reliability of 3D scanner (Artec Eva scanner) in this study. Study 1 investigated the intra-rater reliability ICC $(1,1)$, whereas Study 2 investigated the inter-rater reliability $\operatorname{ICC}(2,1)$.

Results: The intra-rater reliability, surface area 0.98 , and volume ICCS are ICC $0.98,0.98$, and 0.99, respectively, whereas the inter-rater reliability, surface area, and volume ICCS are all 1.00, showing high reliability.

Conclusion: This study suggests that the 3D scanner is a clinically efficient device for measuring the circumference, surface area, and volume of a body part or an object.

\section{INTRODUCTION}

Postoperative orthopedic, heart, and kidney diseases are the focus of rehabilitation medicine. Therefore, swelling and edema should be evaluated quantitatively for the better understanding of disease progression $^{1,2)}$. In general, the method used to measure the circumference is also used to evaluate swelling and edema.
Submitted Jun. 19. 2020

Accepted Sep. 3. 2020

\section{*Correspondence}

Toshihiro Kawae

Makuhari Human Care Faculty Department of Physical Therapy, Tohto University

E-mail: toshikawae@gmail.com

\section{Key words \\ 3D scanner, Reliability, Rehabilitation}

However, if the landmark positions varied due to the therapist's preference, measurement results may presumably be erroneous; therefore, obtaining a reliable clinical evaluation index is difficult. Further, since the circumference measurement is an initial evaluation, the volume of a pathological condition such as edema should be evaluated. Conventionally, evaluation methods using 
magnetic resonance imaging have been investigated for volume measurement; however, this measurement is expensive. Moreover, preparing a measurement environment is difficult and timeconsuming, and thus, it is rarely used in clinical practice ${ }^{3)}$. Although other methods such as water replacement are also accepted to measure volume, they are not widely used in clinical practice because of the need for measurement technology and due to timeconsuming problems ${ }^{4}$.

In recent years, volume of $3 \mathrm{D}$ objects is evaluated using a 3D scanner in the industrial field. In addition, this technique is used for a medical treatment and considered for breast imaging in plastic surgery ${ }^{5}$, and therefore, its therapeutic efficacy for chronic subdural hematoma in neurosurgery ${ }^{6}$ and dermatology as well as in the skin cancer diagnostic technology is evaluated ${ }^{7}$. A 3D scanner is reported as an excellent method used to measure body shape changes, particularly for changes of an amputated lower limb in an obese patient $^{8,9)}$. Moreover, in recent years, 3D scanners have become lightweight, and in particular, the Artec Eva-scanner is a lightweight handheld 3D scanner weighing approximately $900 \mathrm{~g}$ only. Therefore, the volume of edema and situations. However, there are few studies using 3D scanners for the measurement of circumference, area, and volume, yet it is not a clinically established measurement method.

Although volume measurement of the amputated lower limb using this model was assumed to be highly accurate ${ }^{9)}$, the circumference, surface area, and volume of the upper limb and uncut lower limb model were still used by the examiner. However, its intra- and inter-rater reliability has not yet been verified. Therefore, verification of intra- and inter-rater reliability of circumference, surface area, and volume evaluation using a 3D scanner in this study has been considered to show the possibility of being used as a new evaluation rehabilitation method. This study aimed to clarify the reliability of using the Artec Eva scanner in measuring the circumference, surface area, and volume of the upper limbs and various objects.

\section{Participants and Materials}

For the intra-rater reliability of the 3D scanner (Study 1), three healthy male participants (Participant A:age, 23 years; height, 170 cm; weight, $59 \mathrm{~kg}$, and body mass index [BMI], $20.4 \mathrm{~kg} / \mathrm{m}^{2}$; Participant B:28 years, $180 \mathrm{~cm}, 80 \mathrm{~kg}$, and $24.7 \mathrm{~kg} / \mathrm{m}^{2}$; and Participant C:25 years, $172 \mathrm{~cm}, 58 \mathrm{~kg}$, and $19.6 \mathrm{~kg} / \mathrm{m}^{2}$ ) without cardiac or renal disease expected to cause edema and without history of orthopedic disease such as upper limb deformity were recruited.

For the inter-rater reliability of the 3D scanner (Study 2), one leg of the mannequin (Object A) and two character dolls (Objects $\mathrm{B}$ and C) were used.

This study was approved by the Ethical Review Board of Hiroshima University Epidemiological (Approval Number: E-210). All participants were explained of the study protocol and provided informed consent.

\section{Methods}

In this study, the Artec Eva-scanner (Artec 3D Inc., Luxembourg) was used in both Study 1 and Study 2 (Figure 1-A).

In Study 1, the examiner was a female occupational therapist who thoroughly practiced 3D scanner measurement before the actual measurement. After placing the participants in supine position on the bed, the left shoulder joint was flexed at $90^{\circ}$ and the entire upper left limb was measured using the 3D scanner. The examiner held the 3D scanner using the dominant hand over the entire upper limb to be photographed while keeping a distance of $40-60 \mathrm{~cm}$ from the measurement target. The measurement was performed thrice for each participant (Figure 1-B).

Two male physiotherapists (Examiner A, Examiner B) and one female occupational therapist (Examiner C) performed the 3D scanning, and each thoroughly practiced before the actual measurement. Each examiner measured the objects thrice using a 3D scanner.

\section{Data analysis}

In Study 1, after measuring the upper limbs with a 3D scanner, the forearm circumference, forearm surface, and forearm volume were calculated. The forearm circumference was calculated from the plane that passes through the olecranon, olecranon fossa, and lateral epicondyle (Plane 1), and the plane was translated $5 \mathrm{~cm}$ distally (Plane 2). The forearm surface was measured from Plane 2 to a 


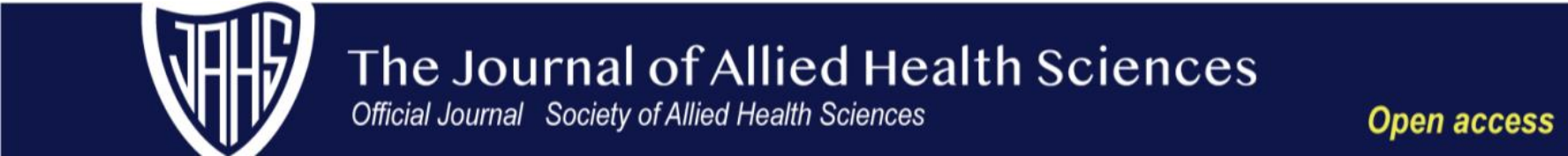

plane passing through two points on the palmar wrist crease and one point on the dorsal wrist crease (Plane 3). The forearm volume was calculated based on the sites used to calculate the forearm surface area (Figure 2-A).

In Study 2, after converting the measured Objects $\mathrm{A}$ to $\mathrm{C}$ into 3D model, the circumference, surface area, and volume were calculated (Figure 2-B), the circumference of Object A was measured in Plane 1, and the surface area and volume were calculated from Planes 1 to 2 . The circumference, surface area, and volume of the entire nose in Object B were calculated in Plane 3. The circumference of Object $\mathrm{C}$ was evaluated in Plane 5, and the surface area and volume were calculated from Planes 4 to 5 . The objects were converted to 3D using Leios2 (E.G.S.s.r.l, Inc., Italy), and the circumference, volume, and surface area were calculated.

Study 1 calculated the standard error of measurement (SEM) for the results three times for each participant, and Study 2 calculated the SEM for measurement results of 3 examiners for each object.

Intra-class correlation coefficients (ICC) were used to evaluate the reliability: ICC $(1,1)$ for intra-rater reliability in Study 1 and ICC $(2,1)$ for inter-rater reliability in Study 2. Furthermore, ICC criteria are $0.00-0.20$ (slight), $0.21-0.40$ (fair), $0.41-0.60$ (moderate), 0.61-0.80 (substantial), and 0.81-1.00 (almost perfect ${ }^{10)}$. The JMP ${ }^{\circledR}$ version 14 (SAS Institute Inc., Cary, NC, USA) was used for statistical analysis.
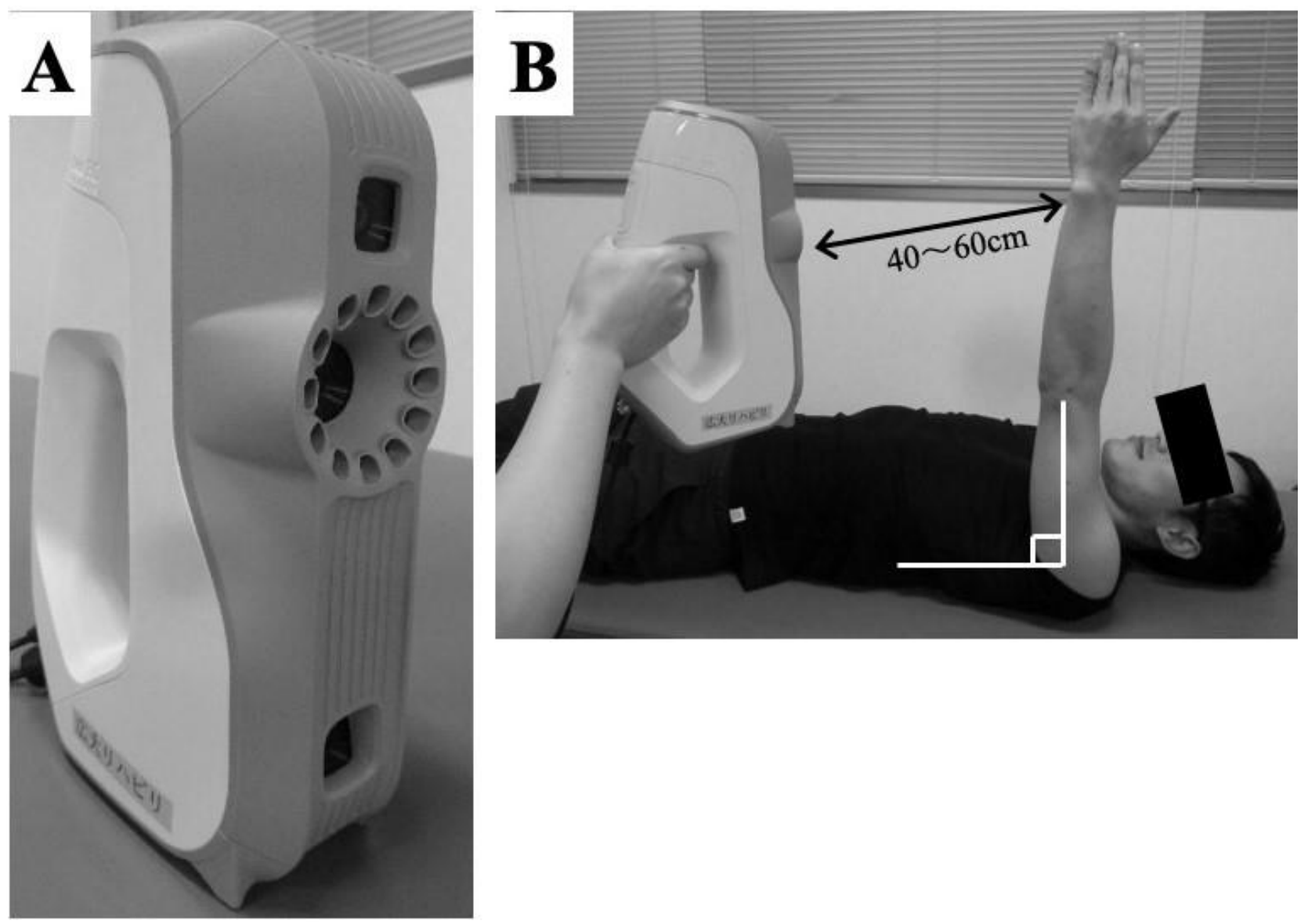

Figure 1

A: The 3D scanner Artec Eva-scanner used in this study (Artec 3D Inc., Luxembourg)

B: Measurement scene in Study 1 


\section{Study1:A}

Study2:B

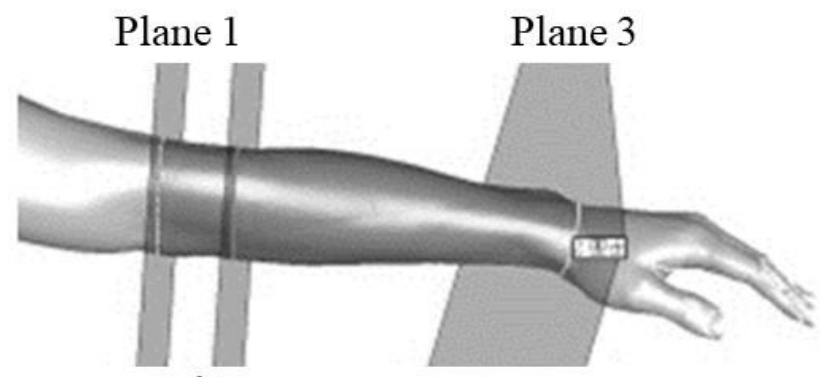

Plane 2
Object A

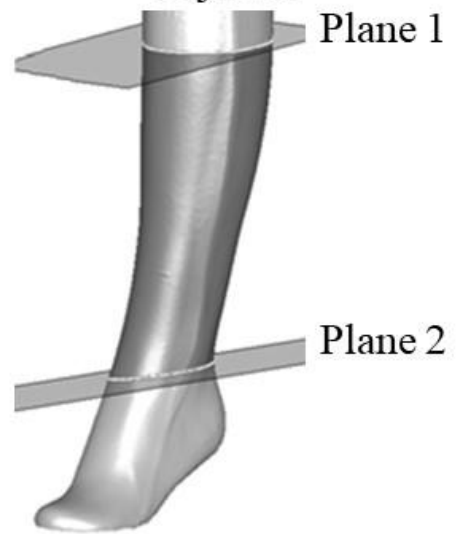

Object B

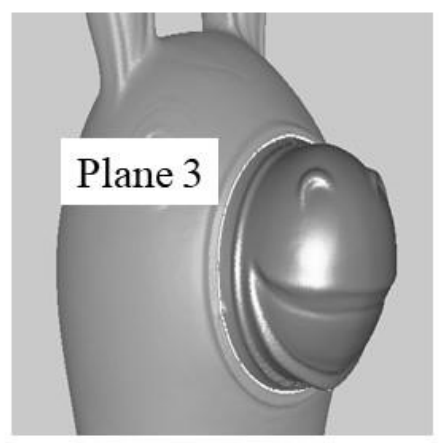

Object C



Figure2

Figure 2 3D image and analysis plane in Study 1 and 2

Study1: Plane 1 (passes through the olecranon, olecranon fossa, and lateral epicondyle), Plane 2 (plane translated from Plane 1 to $5 \mathrm{~cm}$ distally), Plane 3 (a plane that passes through two points on the palmar wrist crease and one point on the dorsal wrist crease)

Study2: Object A (circumference: Plane 1, surface area: from Plane 1 to Plane 2, Volume: from Plane 1 to Plane 2), Object B (circumference: Plane 3, surface area and volume: from Plane 3 to the entire nose), Object C (circumference: Plane 5, surface area: from Plane 4 to Plane 5, volume: from Plane 4 to Plane 5)

\section{RESULTS}

Both Study 1 and 2 were completed in $<1$ min using the $3 \mathrm{D}$ scanner.

In Study 1, the SEM for each participant was $0.1 \mathrm{~cm}$ in circumference, with surface area of $3.6,5.5$, and $1.0 \mathrm{~cm}^{2}$ and volumes of $6.1,11.6$, and $1.1 \mathrm{~cm}^{3}$, respectively. Furthermore, the intra-rater reliability ICC is 0.98 (95\% confidence interval [CI]: $0.89-1.00)$ for the circumference, 0.98 (95\% CI: 0.89-1.00) for the surface area, and 0.99 (95\% CI: 0.94-1.00) for the volume, which showed high reliability (Table 1).

The SEM for each object in Study 2 was $0.3,1.9$, and $0.2 \mathrm{~cm}$ for the circumference; $0.0,0.1$, and $0.0 \mathrm{~cm}^{2}$ for the surface area; and $0.3,1.7$, and $0.1 \mathrm{~cm}^{3}$ for the volume, respectively. Furthermore, the inter-rater reliability was measured once by three investigators. ICC of the circumference, surface area, and volume is calculated, with 1.00 (95\% CI: 0.99-1.00), 1.00 (95\% CI: 1.00-1.00), and 1.00 (95\% CI: 1.00-1.00), respectively, which showed high reliability (Table 2). 
Table 1: Intra-rater reliability for 3D scanners

\begin{tabular}{|c|c|c|c|c|c|}
\hline & \multicolumn{5}{|c|}{ Forearm circumference $(\mathrm{cm})$} \\
\hline & $\operatorname{ICC}(1,1)$ & $1 \mathrm{st}$ & 2nd & $3 \mathrm{rd}$ & SEM \\
\hline Participant A & & 24.7 & 24.5 & 24.5 & 0.1 \\
\hline Participant B & 0.98 & 26.7 & 26.6 & 26.5 & 0.1 \\
\hline \multirow[t]{2}{*}{ Participant C } & & 26.6 & 26.7 & 26.3 & 0.1 \\
\hline & \multicolumn{5}{|c|}{ Surface area of the forearm $\left(\mathrm{cm}^{2}\right)$} \\
\hline Participant A & & 601.3 & 590.2 & 591.0 & 3.6 \\
\hline Participant B & 0.98 & 646.0 & 635.6 & 627.0 & 5.5 \\
\hline \multirow[t]{2}{*}{ Participant C } & & 699.2 & 699.0 & 696.0 & 1.0 \\
\hline & \multicolumn{5}{|c|}{ Forearm volume $\left(\mathrm{cm}^{3}\right)$} \\
\hline Participant A & & 863.7 & 845.8 & 845.1 & 6.1 \\
\hline Participant B & 0.99 & 969.5 & 950.5 & 929.4 & 11.6 \\
\hline Participant C & & 1119.6 & 1122.5 & 1118.8 & 1.1 \\
\hline
\end{tabular}

ICC: Intraclass correlation coefficients $(1,1)$, SEM: Standard error of measurement

Table $2:$ Inter-rater reliability for 3D scanners

\begin{tabular}{|c|c|c|c|c|c|}
\hline \multicolumn{6}{|c|}{ Circumference (cm) } \\
\hline & $\operatorname{ICC}(2,1)$ & Examiner A & Examiner B & Examiner $\mathrm{C}$ & SEM \\
\hline Object A & & 36.3 & 36.3 & 36.3 & 0.0 \\
\hline Object B & 1.00 & 31.2 & 31.2 & 31.5 & 0.1 \\
\hline Object $\mathrm{C}$ & & 12.0 & 12.0 & 12.0 & 0.0 \\
\hline \multicolumn{6}{|c|}{ Surface area $\left(\mathrm{cm}^{2}\right)$} \\
\hline Object A & & 955.7 & 955.4 & 955.5 & 0.1 \\
\hline Object B & 1.00 & 273.9 & 272.6 & 277.9 & 1.6 \\
\hline Object C & & 66.2 & 66.3 & 65.9 & 0.1 \\
\hline \multicolumn{6}{|c|}{ Volume $\left(\mathrm{cm}^{3}\right)$} \\
\hline Object A & & 1869.1 & 1867.9 & 1868.4 & 0.3 \\
\hline Object B & 1.00 & 333.7 & 332.1 & 337.9 & 1.7 \\
\hline Object $\mathrm{C}$ & & 40.6 & 40.8 & 40.3 & 0.1 \\
\hline
\end{tabular}

ICC: Intraclass correlation coefficients $(2,1)$, SEM: Standard error of measurement 


\section{DISCUSSION}

This study verified the reliability of using a 3D scanner, i.e., the Artec Eva-scanner, to measure the circumference, surface area, and volume of the upper limbs and objects. As a result, intra- and inter-rater reliability were almost perfect in Study 1 and 2. During rehabilitation, the circumference, surface area, and volume should be measured accurately. Although volume is a necessary factor to monitor the body water content, accurate measurement requires a special equipment and is clinically important. However, its measurement is costly and requires a special environment. Therefore, in the current clinical practice, a 3D scanner is used as an alternative method to evaluate volume by measuring the weight and using the lower leg circumference ${ }^{11,12)}$. However, to measure the circumference, landmarks cannot be accurately determined, and weight measurement also involves food and water intake. Therefore, evaluation of edema and swelling remains inaccurate. Conversely, the 3D scanner used in this study can measure the volume in $<1 \mathrm{~min}$; therefore, a measuring instrument that can easily evaluate the surface area and volume is suggested. Furthermore, surface area measurements are of great clinical importance, and the Burn Index (BI) is used to measure severity in burn patients. BI was devised to determine burn severity and was calculated as $1 / 2 \times$ area of the second-degree burn (\%) + area of the third-degree burn (\%). A BI of $10-15$ or higher is considered severe ${ }^{13)}$. However, BI uses the rule of nines to calculate the burn area, not the actual measured burn surface area. The rule of nines is a simple method to evaluate the burned area and express all body compartments in multiples of 9. It is also mainly used to assess the burn area of adult patients: the head, 9\%; the upper limbs, 9\% each; the lower limbs, $18 \%$ each; the trunk, $18 \%$; and the pubic area, $1 \%{ }^{14)}$. Therefore, a more accurate $\mathrm{BI}$ can estimated by measuring the burned area with a 3D scanner. In addition, due to the risk of infection through physical contact with a burn patient, a non-contact type 3D scanner can be efficiently used to evaluate the circumference, surface area, and volume to prevent infection.

In this study, the circumference, surface area, and volume were measured using the Artec Eva-scanner, which showed high intra- and inter-rater reliability. Conversely, to verify the intra-rater reliability, SEM of Participant B was found to show high surface area and volume values. SEM is predicted to vary due to different BMIs of participants. Moreover, only Participant B required higher measurement costs among the three participants. Conversely, according to a previous study, a high-performance stationary-type scanner with 32 cameras and 16 sensors with high measurement accuracy does not affect the measurement accuracy even if the waist circumference is high ${ }^{15}$. Therefore, increasing SEM of the surface area and volume due to the use of a handheld scanner is presumed to be an instrumental limit; however, this was not clarified in this study. Therefore, further research is needed on the measurement accuracy for participants with high BMI.

One of the limitations of the present study is that it involved only healthy subjects and objects. In actual clinical practice, subcutaneous tumors and swellings are evaluated. Therefore, it is considered necessary to verify the relationship with more accurate MRI for actual tumors and swelling in the future.

In conclusion, the Artec Eva scanner used in this study was highly reliable in measuring the circumference, surface area, and volume of the body or objects; therefore, it can be considered a clinically applicable device.

\section{Acknowledgment}

We would like to express our sincere gratitude to Kanako Yoshimura, an occupational therapist who has been instrumental in performing the measurements in this study.

\section{References}

1) Guex JJ, Enrici E, Boussetta S, et al.: Correlations between ankle circumference, symptoms, and quality of life demonstrate the clinical relevance of minimal leg swelling reduction: results of a study in 1,036 Argentinean patients. Dermatol Surg 34: 1666-1675, 2008.

2) Ishida $Y$, Maeda $K$, Nonogaki $T$, et al.: Impact of edema on length of calf circumference in older adults. Geriatrics \& Gerontology International 19: 993-998, 2019.

3) Chien MY, Huang TY, Wu YT: Prevalence of sarcopenia estimated using a bioelectrical impedance analysis prediction equation in community-dwelling elderly people in Taiwan. $\mathrm{J}$ Am Geriatr Soc 56: 1710-1715, 2008. 
4) Houska CL, Kemp JD, Niles JS, et al.: Comparison of body composition measurements in lean female athletes. Int $\mathrm{J}$ Exerc Sci 11: 417-424, 2018.

5) Koban KC, Härtnagl F, Titze V, et al.: Chances and limitations of a low-cost mobile 3D scanner for breast imaging in comparison to an established 3D photogrammetric system. $\mathrm{J}$ Plast Reconstr Aesthet Surg 71: 1417-1423, 2018.

6) Bow H, Yang X, Chotai S, et al.: Initial experience with using a structured light 3D scanner and image registration to plan bedside subdural evacuating port system placement. World Neurosurg 137: 350-356, 2020

7) Rey-Barroso L, Burgos-Fernández FJ, Ares $\mathrm{M}$, et al.: Morphological study of skin cancer lesions through a 3D scanner based on fringe projection and machine learning. Biomed Opt Express 10: 3404-3409, 2019.

8) Kroh A, Peters F, Alizai PH, et al.: 3D optical imaging as a new tool for the objective evaluation of body shape changes after bariatric surgery. Obes Surg 30: 1866-1873, 2020.

9) Seminati E, Canepa Talamas D, Young M, et al.: Validity and reliability of a novel 3D scanner for assessment of the shape and volume of amputees' residual limb models. PLoS One 12: e0184498, 2017.

10) Landis JR, Koch GG: The measurement of observer agreement for categorical data. Biometrics 33: 159-174, 1977.

11) Ishida $Y$, Maeda $K$, Nonogaki $T$, et al.: Impact of edema on length of calf circumference in older adults. Geriatr Gerontol Int 19: 993-998, 2019.

12) Webel AR, Frazier SK, Moser DK, et al.: Daily variability in dyspnea, edema and body weight in heart failure patients. Eur J Cardiovasc Nurs 6: 60-65, 2007.

13) Yoshino $Y$, Ohtsuka $M$, Kawaguchi $M$, et al.: The wound/burn guidelines - 6: Guidelines for the management of burns. J Dermatol 43: 989-1010, 2016.

14) Waslen GD: Management of outpatient burns. Can Fam Physician 32: 805-808, 1986.

15) Medina-Inojosa J, Somers VK, Ngwa T, et al.: Reliability of a 3D body scanner for anthropometric measurements of central obesity. Obes Open Access 2, 2016. 